\title{
Uric acid enhances PKC-dependent eNOS phosphorylation and mediates cellular ER stress: A mechanism for uric acid-induced endothelial dysfunction
}

\author{
PENG LI ${ }^{1,2}$, LINA ZHANG $^{3}$, MEI ZHANG ${ }^{1}$, CHANGYONG ZHOU ${ }^{2}$ and NAN LIN ${ }^{2}$ \\ ${ }^{1}$ The Key Laboratory of Cardiovascular Remodeling and Function Research, Chinese Ministy of Education \\ and Chinese Ministy of Public Health, Qilu Hospital of Shandong University, Jinan; Departments of ${ }^{2}$ Emergency Room \\ and ${ }^{3}$ Ophthalmology, The Affiliated Hospital of Qingdao University, Qingdao, Shandong, P.R. China
}

Received July 21, 2015; Accepted February 3, 2016

DOI: $10.3892 / \mathrm{ijmm} .2016 .2491$

\begin{abstract}
The mechanism by which hyperuricemia induced-endothelial dysfunction contributes to cardiovascular diseases (CVDs) is not yet fully understood. In the present study, we used uric acid (UA) to trigger endothelial dysfunction in cultured endothelial cells, and investigated the effects of induced reactive oxygen species (ROS) generation, endoplasmic reticulum (ER) stress induction, and the protein kinase $\mathrm{C}$ (PKC)-dependent endothelial nitric oxide synthase (eNOS) signaling pathway. Human umbilical vein endothelial cells (HUVECs) were incubated with 6, 9 or $12 \mathrm{mg} / \mathrm{dl}$ UA, ROS scavenger polyethylene glycol-superoxide dismutase (PEG-SOD), ER stress inhibitor 4-phenylbutyric acid (4-PBA), and PKC inhibitor polymyxin B for 6-48 h. Nitric oxide (NO) production, eNOS activity, intracellular ROS, ER stress levels, and the interaction between eNOS and calmodulin (CaM) and cytosolic calcium levels were assessed using fluorescence microscopy and western blot analysis. Apoptosis was assessed by annexin V staining. UA increased HUVEC apoptosis and reduced eNOS activity and NO production in a dose- and time-dependent manner. Intracellular ROS was elevated after $3 \mathrm{~h}$, while ER stress level increased after $6 \mathrm{~h}$. UA did not alter intracellular $\mathrm{Ca}^{2+}, \mathrm{CaM}$, or eNOS concentration, or eNOS Ser ${ }^{177}$ phosphorylation. However, PKC-dependent eNOS phosphorylation at $\mathrm{Thr}^{495}$ was greatly enhanced, and consequently interaction between eNOS and CaM was reduced. Cellular ROS depletion, ER stress inhibition and PKC activity reduction inhibited the effect of UA on eNOS activity, NO release and apoptosis in HUVECs. Thus, we concluded that UA
\end{abstract}

Correspondence to: Dr Mei Zhang, The Key Laboratory of Cardiovascular Remodeling and Function Research, Chinese Ministy of Education and Chinese Ministy of Public Health, Qilu Hospital of Shandong University, Jinan, Shandong 250012, P.R. China E-mail: zhangmei718866@163.com

Key words: uric acid, endothelial cells, reactive oxygen species, endoplasmic reticulum stress, endothelial nitric oxide synthase, protein kinase $\mathrm{C}$ induced HUVEC apoptosis and endothelial dysfunction by triggering oxidative and ER stress through PKC/eNOS-mediated eNOS activity and NO production.

\section{Introduction}

Uric acid (UA) is the end product of purine metabolism in humans and higher primates, and humans tend to have higher blood UA levels than other mammals (1). Pathological accumulation of UA in the blood (hyperuricemia) is associated with gout, lithangiuria, and UA nephropathy. Hyperuricemia has recently been reported to be a risk factor for cardiovascular diseases (CVDs), chronic kidney diseases (CKDs), hypertension and kidney disease (1-6), but the mechanisms are not yet fully understood.

The relatively high physiological level of UA in humans has been suggested to play an antioxidative defense role $(7,8)$, but previous research has reported that higher levels of UA trigger oxidative stress in cells, and contribute to endothelial dysfunction (9). High concentrations of UA enhance reactive oxygen species (ROS) generation in neutrophils (10), and UA induced oxidative stress and inhibited growth of pancreatic $\beta$-cells (11). In addition, in endothelial cells, hyperuricemia has been reported to trigger cellular oxidative stress and endothelial dysfunction (12).

Cellular stresses trigger endoplasmic reticulum (ER) stress, which activates a complex signaling network, known as the unfolded protein response (UPR), to reduce ER stress and restore homeostasis (13), but sustained and unresolvable ER stress promotes apoptosis, elevates CCAAT-enhancer-binding protein homologous protein $(\mathrm{CHOP})$ and activates transcription factor 6 (ATF-6) expression, inducing endothelial dysfunction (14-16). ER stress also activates protein kinase C (PKC) and AKT signaling pathways (17-19). UA was reported to induce ER stress in glomerular mesangial cells and hepatocytes $(20,21)$.

Endothelial dysfunction is characterized by decreased nitric oxide (NO) levels and cellular activity, and contributes to the pathogenesis of CVD. Hyperuricemia has been reported to induce endothelial dysfunction in several studies $(6,9,22,23)$. Allopurinol therapy, which reduces UA levels, has been noted to improve endothelial function $(24,25)$. In endothelial cells, a high concentration of UA increased ROS levels, and decreased eNOS activity and NO production $(22,26)$. One possible 
mechanism involves the phosphorylation of eNOS $(27,28)$. In renal proximal tubule cells, UA has been reported to inhibit proliferation through the PKC signaling pathway (29). UA does not alter eNOS expression in human umbilical vein endothelial cells (HUVECs), but abolished the interaction of eNOS with calmodulin $(\mathrm{CaM})$ and NO release (22).

Therefore, in the present study, we investigated the effect of UA on eNOS phosphorylation, eNOS activity, and NO production in primary culture HUVECs. We detected ER stress in HUVECs incubated with UA. We noted that UA triggers oxidative and ER stress through PKC/eNOS-mediated eNOS activity and NO production, causing HUVEC apoptosis.

\section{Materials and methods}

Reagents. UA ( $>99 \%$ pure, without endotoxin), polyethylene glycol-superoxide dismutase (PEG-SOD), 4-phenylbutyric acid (4-PBA), and polymyxin B were purchased from SigmaAldrich (St. Louis, MO, USA). Fluo-3, acetoxymethyl (AM) was purchased from Molecular Probes, Inc. (Eugene, OR, USA). Antibodies against p-eNOS-Thr ${ }^{495}$ (ab138430), p-eNOS-Ser ${ }^{177}$ (ab195944), eNOS (ab66127), caspase-12 (ab62484), CHOP (ab11419), and ATF-6 (ab11909) were obtained from Abcam (Cambridge, UK). Primary monoclonal antibody against human CaM (05-173) was from Millipore Corp. (Billerica, MA, USA). Primary polyclonal antibodies against PKC (\#2056) and p-PKC (\#9375) and HRP-linked secondary antibodies against rabbit IgG (\#7074), mouse IgG (\#7076) were purchased from Cell Signaling Technology, Inc. (Boston, MA, USA). The Annexin V-FITC Apoptosis Detection kit was from BD Pharmingen (San Diego, CA, USA), and the Nitric Oxide Synthase Assay kit was from Beyotime Biotech (Jiangsu, China). All chemicals were of analytical grade.

HUVEC culture. Primary HUVECs were purchased from PromoCell (Heidelberg, Germany) and cultured at $37^{\circ} \mathrm{C}$ in $5 \% \mathrm{CO}_{2}$ in Endothelial Cell Growth Medium (Cat. no. C-22010; PromoCell), which contains 2\% fetal bovine serum, $5 \mathrm{ng} /$ $\mathrm{ml}$ epidermal growth factor (EGF), $10 \mathrm{ng} / \mathrm{ml}$ basic fibroblast growth factor (bFGF), $20 \mathrm{ng} / \mathrm{ml}$ Long R3 IGF-1, and $0.5 \mathrm{ng}$ / $\mathrm{ml}$ vascular endothelial growth factor (VEGF). Cells at passage 3-7 were used for experiments.

UA solution. The UA solution was prepared as follows: $0.4 \mathrm{~g}$ of $\mathrm{NaOH}$ was dissolved in $10 \mathrm{ml}$ ultrapure water to obtain $1 \mathrm{mM}$ $\mathrm{NaOH}$ solution. Subsequently, $150 \mathrm{mg}$ UA was added, fully dissolved in a warm water bath under agitation. The final UA concentration was $15 \mathrm{mg} / \mathrm{ml}$. The solution was filtered using a $0.22-\mu \mathrm{m}$ filter under a sterile hood for eventual removal of microorganisms. The solution was stored at room temperature. Prior to the experiment, the stock solution was used to obtained culture media at 6,9 and $12 \mathrm{mg} / \mathrm{dl}$.

UA stimulation and pharmacological inhibition. UA was added to HUVEC cultures $24 \mathrm{~h}$ after passage, at final concentrations of 6,9 or $12 \mathrm{mg} / \mathrm{dl}$. Where indicated, $30 \mathrm{~min}$ prior to the addition of UA, the following inhibitors were added at the indicated final concentrations: $100 \mathrm{U} / \mathrm{ml}$ of the PEG-SOD antioxidant, $10 \mathrm{mM}$ of the ER stress inhibitor 4-PBA, or $20 \mu \mathrm{g} / \mathrm{ml}$ of the PKC inhibitor polymyxin B. Untreated HUVECs were used as the control.
Analysis of apoptosis using flow cytometry. Trypsin-dispersed HUVECs $\left(5 \times 10^{4} / \mathrm{sample}\right)$ were resuspended in $200 \mu \mathrm{l}$ Annexin V-FITC and $10 \mu \mathrm{l}$ propidium iodide and subsequently incubated at room temperature $\left(20-25^{\circ} \mathrm{C}\right)$ in the dark for $20 \mathrm{~min}$. Staining was then analyzed using a Becton-Dickinson flow cytometer (Becton-Dickinson, San Diego, CA, USA).

Measurement of NO release. Cell culture supernatant NO content was measured using a Total Nitric Oxide Assay kit (nitrate/nitrite assay kit; Beyotime Biotech) according to the manufacturer's instructions. Total NO production was estimated according to the accumulation of nitrite and nitrate in the cell culture supernatant using Griess reagent (Beyotime Biotech). Nitrate was converted enzymatically into nitrite by nitrate reductase. Nitrite is a stable metabolite of $\mathrm{NO}$, and thus is an indicator of released NO in the media, as previously described (30).

Measurement of eNOS activity. eNOS activity was measured using a Nitric Oxide Synthase Assay kit (Beyotime Biotech) according to the manufacturer's instructions. As previously described (31), HUVECs were seeded in a 96-well plate. Cells were resuspended in $100 \mu \mathrm{l}$ NOS detection buffer and $100 \mu \mathrm{l}$ reaction buffercontaining NOS substrates $(5 \mu 10.1 \mathrm{mMNADPH}$ and L-arginine, respectively) and a cell-permeable fluorescent substance (5 $\mu 1$ 3-amino 14-aminomethyl-2',7'-difluorescein diacetate) and incubated at $37^{\circ} \mathrm{C}$ in the dark for $40 \mathrm{~min}$. Fluorescence intensity (FI) was measured at Ex $495 \mathrm{~nm} /$ Em $515 \mathrm{~nm}$ on a microplate reader (Molecular Devices, LLC, Sunnyvale, CA, USA). The relative activity (RA) of eNOS was calculated as:

$$
\mathrm{RA}=\frac{\mathrm{FI}(\text { sample) }-\mathrm{FI} \text { (negative) }}{\mathrm{FI}(\text { control) }-\mathrm{FI} \text { (negative) }}
$$

Measurement of ROS. The intracellular ROS levels were measured using CM-H2DCFDA (Sigma, Carlsbad, CA, USA). HUVECs were seeded on $35-\mathrm{mm}$ confocal culture dishes at $4 \times 10^{4}$ cells $/ \mathrm{ml}$ in $2 \mathrm{ml}$ media per dish $24 \mathrm{~h}$ before UA stimulation. After stimulation, culture media was replaced with dichloro-dihydro-fluorescein diacetate (DCFH-DA), diluted in serum-free media to a final concentration of $10 \mu \mathrm{M}$, and cells were incubated at $37^{\circ} \mathrm{C}$ in the dark. After $30 \mathrm{~min}$, the cells were washed with serum-free media three times and FI was measured using a Nikon A1 confocal microscope (purchased from Nikon Instruments, Inc., Tokyo, Japan) at Ex $488 \mathrm{~nm} / \mathrm{Em} 515 \mathrm{~nm}$.

Measurement of $\mathrm{Ca}^{2+}$ concentration. The intracellular level of $\mathrm{Ca}^{2+}$ was measured using the $\mathrm{Ca}^{2+}$-specific fluorescent probe Fluo-3, AM, as previously described (26). HUVECs were cultured in the presence or absence of $12 \mathrm{mg} / \mathrm{dl}$ of UA for 6 , 12 or $24 \mathrm{~h}$, then incubated with $50 \mu \mathrm{M}$ Fluo- $3, \mathrm{AM}$ at $37^{\circ} \mathrm{C}$ in the dark. After $1 \mathrm{~h}$, cells were washed three times in Hanks' solution to remove unloaded probes. The FI of Fluo-3, AM was measured at Ex $488 \mathrm{~nm} / \mathrm{Em} 525 \mathrm{~nm}$ using a Nikon A1 confocal microscope (Nikon Instruments, Inc.).

Co-immunoprecipitation assay. HUVECs were harvested with cell lysis buffer containing protease inhibitors (\#5871; Cell Signaling Technology, Inc., Danvers, MA, USA) on ice for 

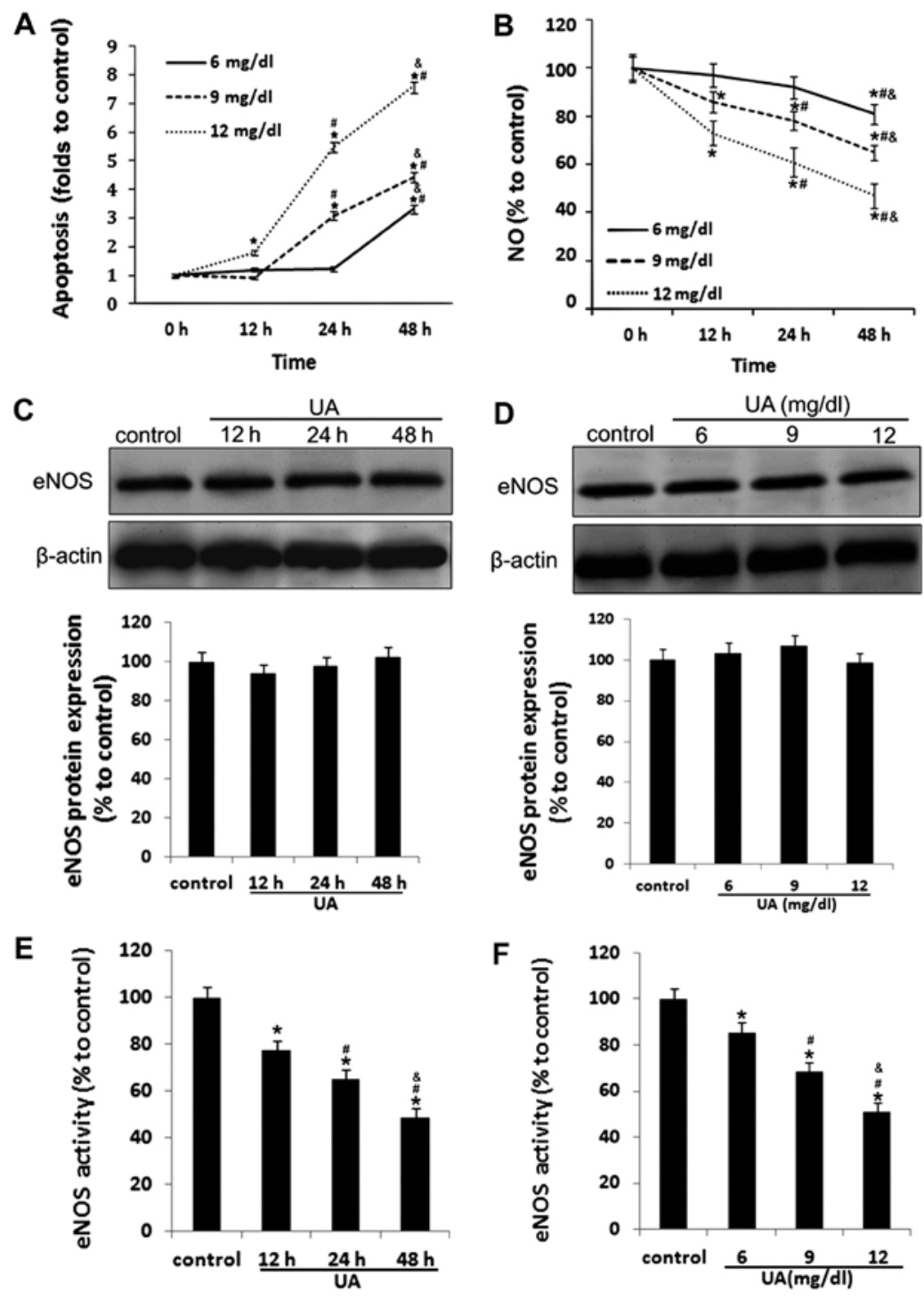

Figure 1. Uric acid (UA) induces apoptosis and decreases nitric oxide (NO) release and endothelial nitric oxide synthase (eNOS) activity in human umbilical vein endothelial cells (HUVECs). Primary cultured HUVECs were incubated with 6,9 and $12 \mathrm{mg} / \mathrm{dl}$ of UA for the indicated periods of time. (A) Apoptosis was measured by Annexin V/PI staining, and detected by flow cytometry. (B) NO release was measured using Griess reagent. (C and D) eNOS protein levels were measured by western blot analysis in HUVECs incubated with $12 \mathrm{mg} / \mathrm{dl}$ UA. (E and F) eNOS activity was measured using a NOS activity assay kit. ${ }^{*} \mathrm{P}<0.05$ vs. control; ${ }^{\#} \mathrm{P}<0.05$ vs. $12 \mathrm{~h}$ and $6 \mathrm{mg} / \mathrm{dl} ;{ }^{~} \mathrm{P}<0.05$ vs. $24 \mathrm{~h}$ and $9 \mathrm{mg} / \mathrm{dl} ; \mathrm{n}=3-5$.

$30 \mathrm{~min}$. The supernatant protein lysates were collected after $30 \mathrm{~min}$ of centrifugation at $1,200 \mathrm{xg}$. Lysate $(500 \mu \mathrm{g})$ was incubated overnight with $3 \mu \mathrm{g}$ eNOS-directed antibody or preimmune IgG (Cell Sciences, Canton, MA, USA) at $4^{\circ} \mathrm{C}$. Labeled lysates were incubated with $25 \mu \mathrm{l}$ of protein A agarose beads (Santa Cruz Biotechnology, Inc., Santa Cruz, CA, USA) at $4^{\circ} \mathrm{C}$. After $3 \mathrm{~h}$, protein A-agarose beads were washed three times with lysis buffer and resuspended in $15 \mu \mathrm{l}$ of $2 \mathrm{X}$ SDS loading buffer, boiled for $5 \mathrm{~min}$, and analyzed by SDS-PAGE and western blot analysis.

Western blot analysis. HUVECs were lysed in RIPA buffer with phosphatase inhibitor cocktail (100X) (\#5870; Cell Signaling Technology, Inc., Danvers, MA, USA) and stored at $-70^{\circ} \mathrm{C}$ after determining the protein concentration using the BCA reagent (Beyotime Biotech). As described previously (32), proteins were separated on SDS-PAGE with a 5\% stacking gel and a $12 \%$ resolving gel. After separation, proteins were electrophoretically transferred onto PVDF membranes (Millipore Corp.), and incubated in blocking buffer (Beyotime Biotech) for $2 \mathrm{~h}$ at room temperature. Blots were probed with primary antibodies (against p-eNOS-Thr495, p-eNOS-Ser1177, eNOS, caspase-12, CHOP, ATF-6, CaM, PKC, p-PKC and $\beta$-actin) overnight at $4^{\circ} \mathrm{C}$, then corresponding HRP-labeled secondary antibodies (against rabbit $\operatorname{IgG}$ or mouse $\operatorname{IgG}$ ), and developed using enhanced chemiluminescence reagents (Millipore Corp.). Band intensity was analyzed using the Touch Gel Dock system (UVP LLC, Upland, CA, USA). $\beta$-actin was used as the internal reference; the relative expression of protein was represented as the ratio of the target protein to $\beta$-actin (target protein $/ \beta$-actin).

Statistical analysis. All data in the present study are presented as the means \pm standard deviation (SD). Statistical analysis was performed using SPSS. Group characteristics were compared 
A
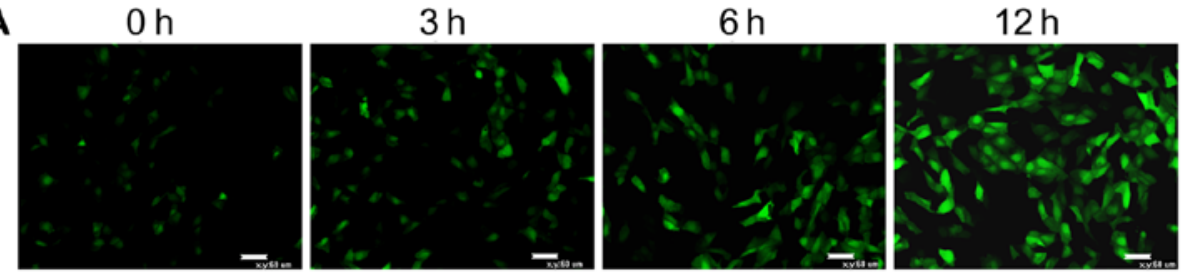

B
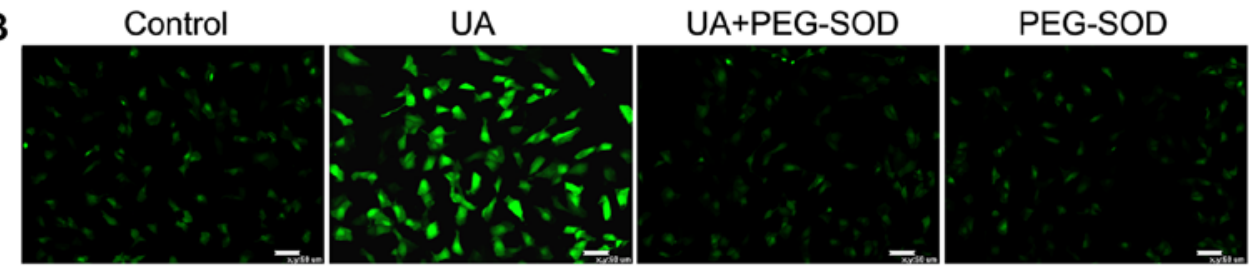

C

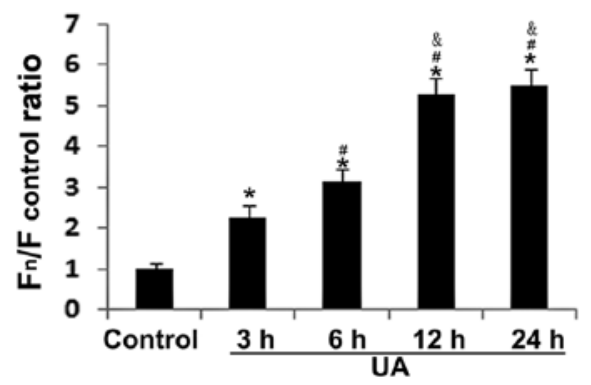

D

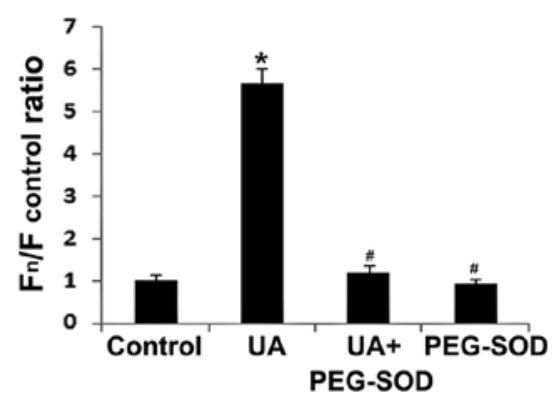

$24 \mathrm{~h}$

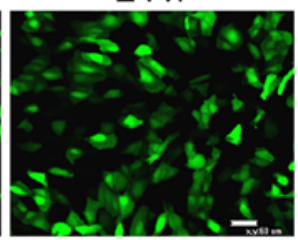

Figure 2. Effect of uric acid (UA) on intracellular reactive oxygen species (ROS) generation in human umbilical vein endothelial cells (HUVECs). (A and C) Intracellular ROS levels were detected using the specific probe CM-H2DCFDA. (B and D) HUVECs were pre-treated with or without 100 U/m1 polyethylene glycol-superoxide dismutase (PEG-SOD) for $30 \mathrm{~min}$ before incubation with $\mathrm{UA}$. " $\mathrm{P}<0.05 \mathrm{vs}$. control; ${ }^{\sharp} \mathrm{P}<0.05 \mathrm{vs} .3 \mathrm{~h}$ and $\mathrm{UA} ;{ }^{\star} \mathrm{P}<0.05 \mathrm{vs} .6 \mathrm{~h} ; \mathrm{n}=3$.

by one-way ANOVA, followed by the Student-NewmanKeuls (SNK-q) post hoc test. A P-value $<0.05$ was considered to indicate a statistically significant difference.

\section{Results}

UA enhances apoptosis and reduces NO production. As shown in Fig. 1A, in the present study we examined the effect of UA on vascular endothelial cellular function and NO production by treating in vitro cultured HUVECs with various concentrations of UA for different durations of time. We found that the rate of apoptosis was increased in HUVECs incubated with UA in a time- and concentration-dependent manner. Significantly increased HUVEC apoptosis was detected within $48 \mathrm{~h}$ in HUVECs incubated with $6 \mathrm{mg} / \mathrm{dl}$ of UA, within $24 \mathrm{~h}$ in HUVECs incubated with $9 \mathrm{mg} / \mathrm{dl}$ of UA, and within $12 \mathrm{~h}$ in HUVECs incubated with $12 \mathrm{mg} / \mathrm{dl}$ of UA (all P<0.05) (Fig. 1A).

Incubation of HUVECs with UA reduced NO production in a time- and concentration-dependent manner (Fig. 1B) and reduced eNOS activity (Fig. 1E and F), whereas even $12 \mathrm{mg} / \mathrm{dl}$ UA barely affected the protein expression of eNOS (Fig. 1C and D).

UA increases intracellular ROS generation. Oxidative stress is known to play an important role in UA-induced endothelial dysfunction $(12,26)$. We measured intracellular ROS levels in order to estimate oxidative stress using a specific probe, CM-H2DCFDA, in primary HUVEC cultures. Intracellular ROS levels were significantly increased $3 \mathrm{~h}$ after incubation with $12 \mathrm{mg} / \mathrm{dl}$ of UA, and remained significantly elevated for $24 \mathrm{~h}(\mathrm{P}<0.05)$ (Fig. 2A and C). Pretreatment of HUVECs with the cell-permeable ROS scavenger PEG-SOD (26) for $30 \mathrm{~min}$ significantly ameliorated the UA-induced increase in intracellular ROS accumulation (Fig. 2B and D).

UA-induced oxidative stress triggers ER stress in HUVECs. UA has previously been reported to regulate cell function through the ER stress-signaling network in glomerular mesangial cells and hepatocytes, and oxidative stress is one of the key drivers of ER stress $(20,21)$. To investigate the role of ER stress in UA-induced endothelial dysfunction, we measured the cellular content of the ER stress biomarkers ATF-6 and CHOP, and the ER stress-induced apoptotic marker caspase-12, by western blot analysis. UA (12 mg/dl) increased the levels of ER stress markers in HUVECs in a time-dependent manner (Fig. 3A). The cellular content of ATF-6 and CHOP was significantly increased after $6 \mathrm{~h}$ of incubation with UA, rose again at $12 \mathrm{~h}$, and remained elevated for $24 \mathrm{~h}$; for $\mathrm{CHOP}$, expression peaked at $12 \mathrm{~h}$ and for ATF- 6 at $24 \mathrm{~h}$. Cellular levels of caspase-12 were significantly increased at $12 \mathrm{~h}$ and peaked at $24 \mathrm{~h}$. HUVECs were pre-treated with the antioxidant PEG-SOD or ER stress inhibitor 4-PBA in order to explore the association between UA-induced oxidative stress and ER stress. Both PEG-SOD and 4-PBA effectively inhibited UA-induced ATF-6, CHOP, and caspase-12 upregulation (Fig. 3B). UA-induced oxidative stress was observed after $3 \mathrm{~h}$, and ER stress was observed after $6 \mathrm{~h}$. These results suggest that UA stimulation induces oxidative stress, which triggers ER stress in HUVECs. 
A

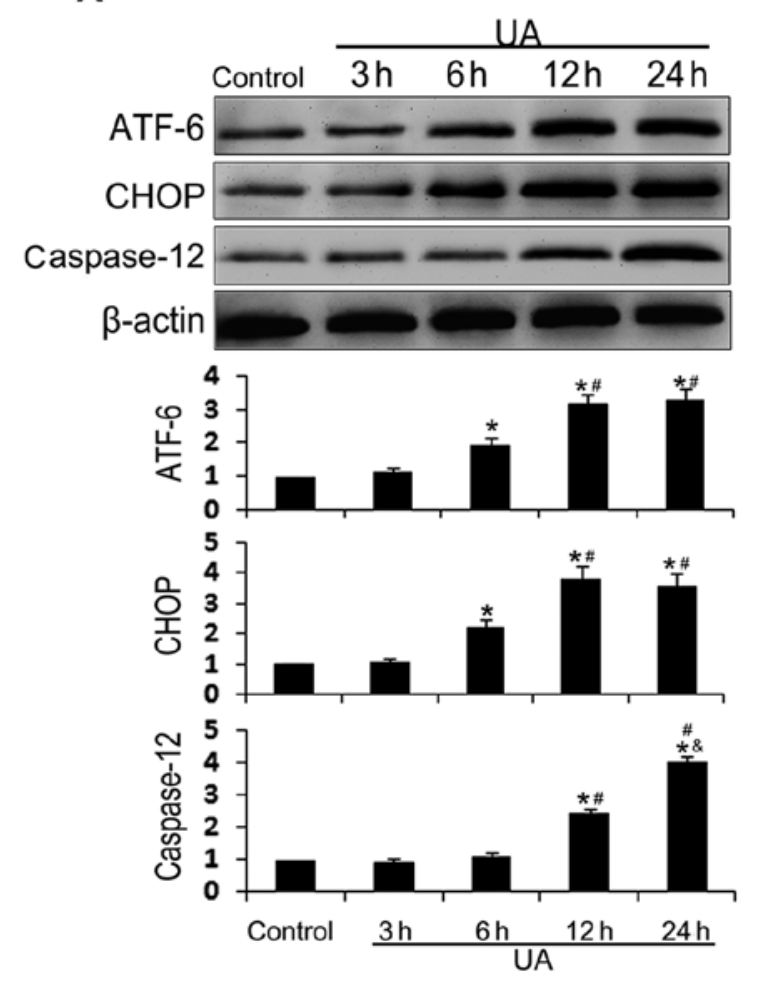

B
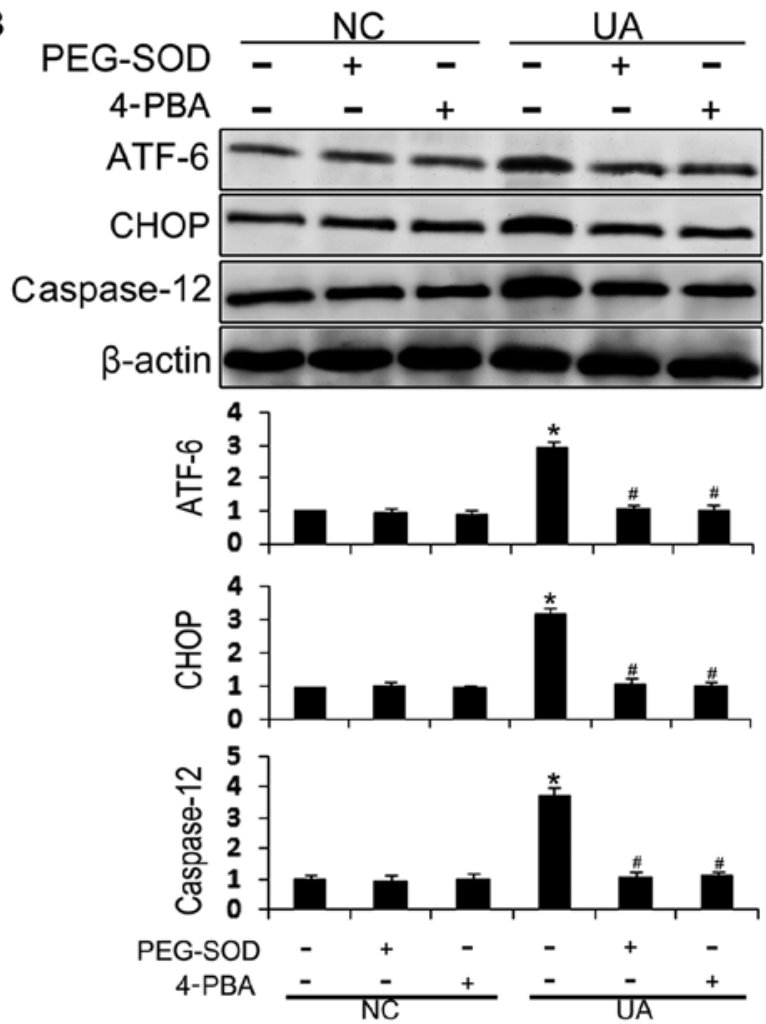

Figure 3. Effect of uric acid (UA) on endoplasmic reticulum (ER) stress in human umbilical vein endothelial cells (HUVECs). (A) HUVECs were incubated with $12 \mathrm{mg} / \mathrm{dl}$ of UA for 3, 6, 12 and $24 \mathrm{~h}$. Activating transcription factor 6 (ATF-6), CCAAT-enhancer-binding protein homologous protein (CHOP), and caspase-12 protein expression was assessed by western blot analysis. (B) HUVECs were incubated with $100 \mathrm{U} / \mathrm{ml}$ of polyethylene glycol-superoxide dismutase (PEG-SOD) or $10 \mathrm{mM}$ of 4-phenylbutyric acid (4-PBA) for $30 \mathrm{~min}$, prior to incubation with or without $\mathrm{UA}$ for $24 \mathrm{~h}$. ${ }^{*} \mathrm{P}<0.05 \mathrm{vs}$. control; ${ }^{\#} \mathrm{P}<0.05 \mathrm{vs}$. $6 \mathrm{~h}$ and $\mathrm{UA} ;{ }^{\&} \mathrm{P}<0.05$ vs. $12 \mathrm{~h} ; \mathrm{n}=3$. NC, HUVECs not stimulated with UA.

ROS scavenging and ER stress inhibition attenuate UA-induced apoptosis and decrease of NO production. To further confirm the roles of oxidative stress and ER stress in UA-induced apoptosis and endothelial dysfunction, we assessed the rate of apoptosis and NO production in HUVECs pre-treated with PEG-SOD and 4-PBA. While HUVECs incubated with $12 \mathrm{mg} / \mathrm{dl}$ UA for $48 \mathrm{~h}$ exhibited increased apoptosis and reduced NO production $(\mathrm{P}<0.05)$, in cells pre-incubated with PEG-SOD or 4-PBA this effect was not observed (Fig. 4), suggesting that UA induces endothelial dysfunction by triggering oxidative stress and ER stress.

UA decreases eNOS activity by increasing Thr ${ }^{495}$ phosphorylation. $\mathrm{Ca}^{2+}, \mathrm{CaM}$, eNOS, and eNOS phosphorylation state are known to act as key regulators of eNOS activity $(22,33)$ and were thus examined in HUVECs in the present study. HUVECs incubated with $12 \mathrm{mg} / \mathrm{dl}$ UA for 6,12 or $24 \mathrm{~h}$ contained higher levels of eNOS and CaM. Although the intracellular $\mathrm{Ca}^{2+}$ concentration, the rate of eNOS phosphorylation at $\mathrm{Ser}^{1177}$, and CaM expression were almost unaltered (Fig. 5A-C), eNOS phosphorylation at $\mathrm{Thr}^{495}$ was increased in a time-dependent manner between 6 and $24 \mathrm{~h}(\mathrm{P}<0.05)$ (Fig. 5A). Binding of eNOS and CaM was assessed by co-immunoprecipitation, and we noted that UA also reduced eNOS and CaM binding in a time-dependent manner $(\mathrm{P}<0.05)$ (Fig. 5D), which was negatively correlated with increased phosphorylation of eNOS at $\mathrm{Thr}^{495}$. These findings indicated that UA inhibits eNOS activity through phosphorylation of eNOS at $\mathrm{Thr}^{495}$ and prohibits its interaction with CaM.

$U A$ decreases eNOS activity through the ER stress/PKC pathway in HUVECs. PKC activates eNOS enzymatic activity by phosphorylating eNOS at $\operatorname{Thr}^{495}$ (28). To further investigate the mechanism by which UA modulates eNOS activity, PKC expression and phosphorylation, HUVECs were incubated with the antioxidant PEG-SOD, the ER stress inhibitor 4-PBA, and the PKC inhibitor polymyxin B prior to incubation with UA. The PKC expression level was almost unaltered by incubation with UA, whereas the level of p-PKC was elevated after $6 \mathrm{~h}$ and peaked at $12 \mathrm{~h}(\mathrm{P}<0.05)$ (Fig. 6A). Moreover, PEG-SOD, 4-PBA, or polymyxin B pretreatment inhibited UA-induced PKC phosphorylation (Fig. 6B-D). These results suggest that UA triggers ER stress and PKC phosphorylation by inducing ROS generation. Active PKC increases phosphorylation of eNOS at $\mathrm{Thr}^{495}$, and reduces the interaction between eNOS and CaM, thus inhibiting eNOS activity and NO production.

\section{Discussion}

In the present study, we sought to determine the mechanisms by which UA induces endothelial dysfunction, and also to investigate the effects of UA on eNOS phosphorylation, eNOS activity, and NO production in primary culture HUVECs. UA increased the rate of apoptosis, and decreased eNOS activity 
A
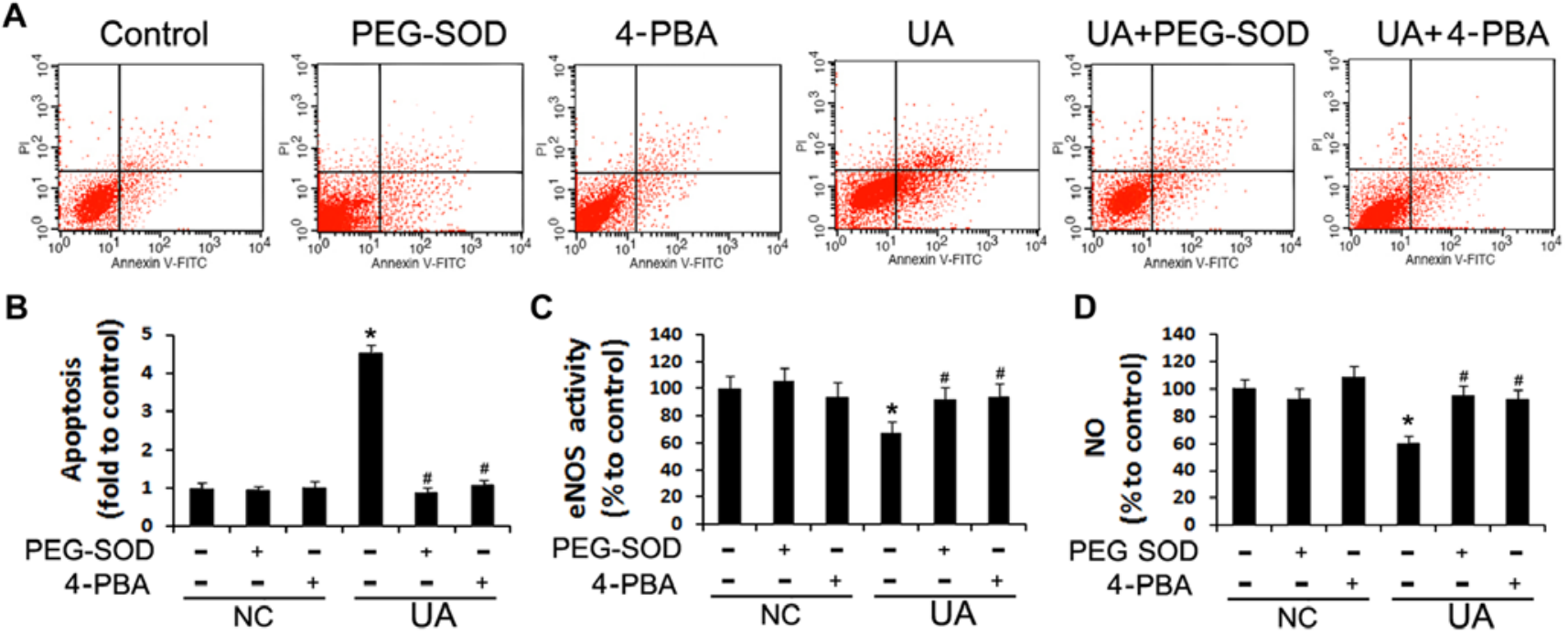

Figure 4. Effect of polyethylene glycol-superoxide dismutase (PEG-SOD) and 4-phenylbutyric acid (4-PBA) on cell apoptosis and nitric oxide (NO) release in human umbilical vein endothelial cells (HUVECs). NC indicates HUVECs which were incubated without UA stimulation, just with different pharmacological pretreatment. (A and B) HUVECs were incubated with $12 \mathrm{mg} / \mathrm{dl}$ of uric acid (UA) with or without a 30-min pre-incubation with PEG-SOD or 4-PBA. HUVEC apoptosis was measured with an Annexin V-FITC kit. (C and D), and endothelial nitric oxide (NO) synthase (eNOS) activity and NO release were measured using a NOS activity assay kit and Griess reagent, respectively. ${ }^{*} \mathrm{P}<0.05$ vs. control; ${ }^{\mathrm{P}} \mathrm{P}<0.05$ vs. UA; $\mathrm{n}=3$. NC, HUVECs not stimulated with UA.
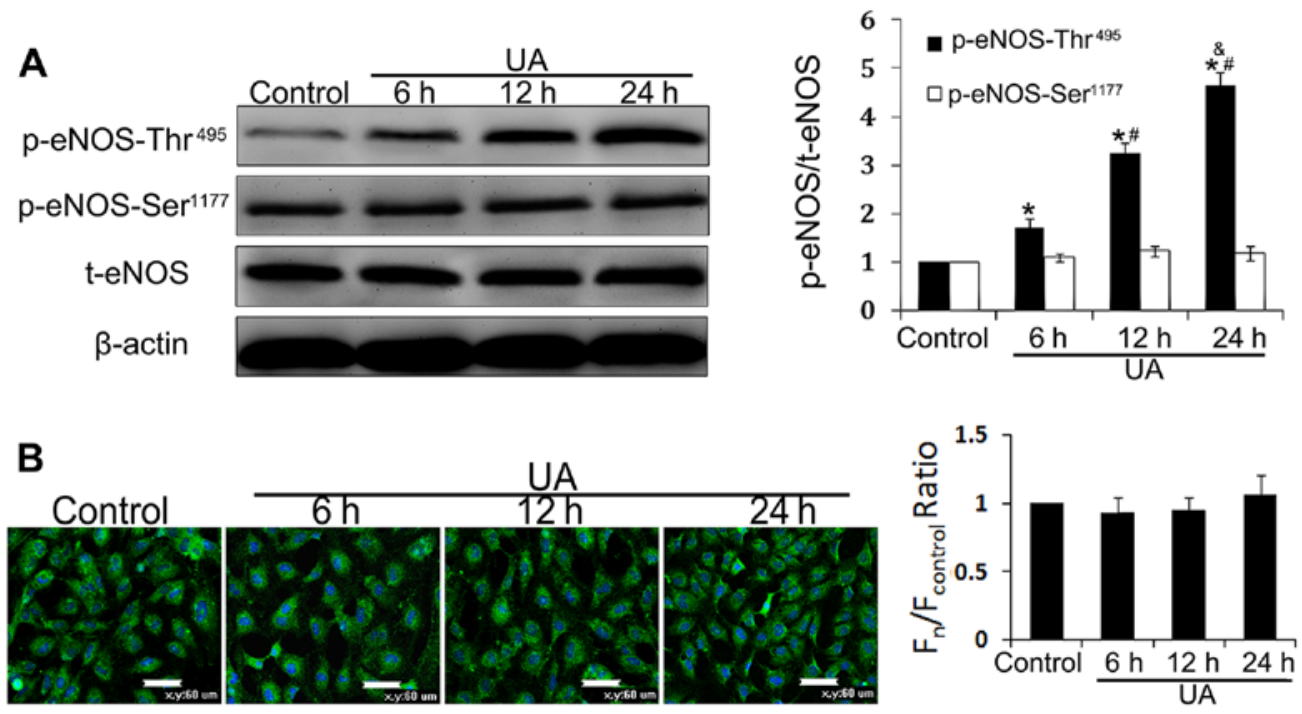

C

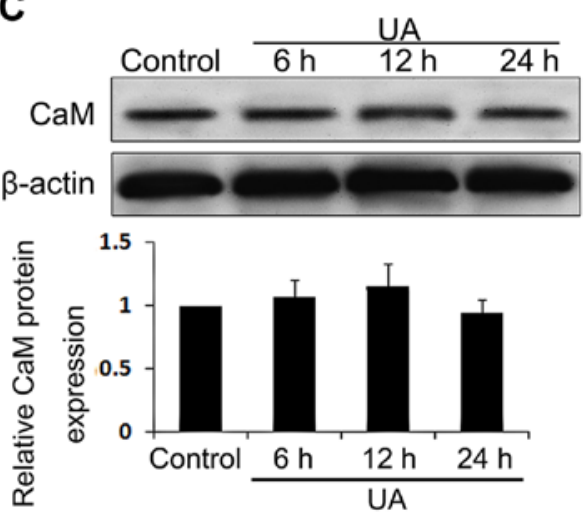

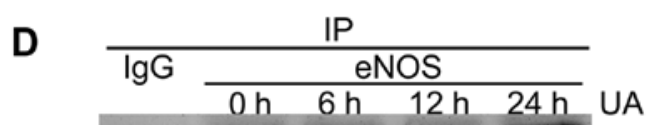

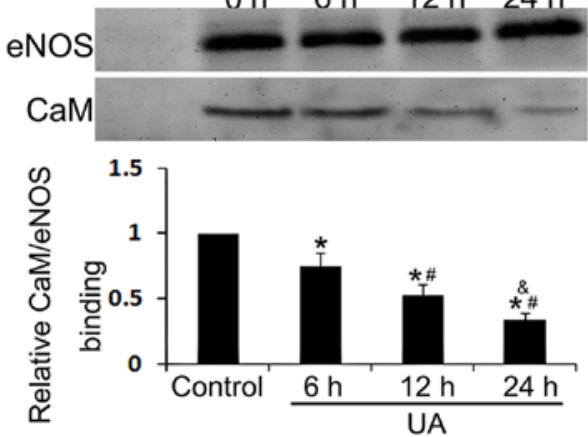

Figure 5. Uric acid (UA) decreases endothelial nitric oxide synthase (eNOS) activity by phosphorylation at Thr ${ }^{495}$. Human umbilical vein endothelial cells (HUVECs) were incubated with $12 \mathrm{mg} / \mathrm{dl}$ of UA for 6, 12 and $24 \mathrm{~h}$. (A) Cellular content of p-eNOS-Ser ${ }^{1777}$, p-eNOS-Thr ${ }^{495}$ and eNOS was assessed by western blot analysis. (B) Intracellular $\left(\mathrm{Ca}^{2+}\right)_{\text {cyto }}$ was assessed using the specific probe Fluo-3, AM by laser confocal microscope. (C) Cellular content of calmodulin (CaM) was assessed by western blot analysis. (D) Co-immunoprecipitation of eNOS and CaM from lysates of UA-treated HUVECs using an eNOS-directed antibody, and detected using a CaM-directed antibody. ${ }^{*} \mathrm{P}<0.05$ vs. control; ${ }^{*} \mathrm{P}<0.05$ vs. $6 \mathrm{~h}$; ${ }^{\circledR} \mathrm{P}<0.05$ vs. $12 \mathrm{~h} ; \mathrm{n}=3$. 
A
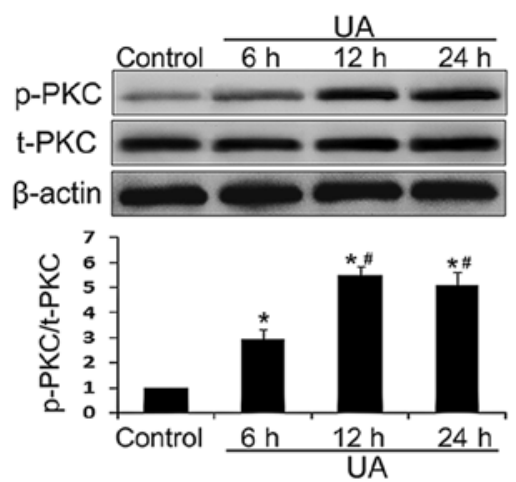

C

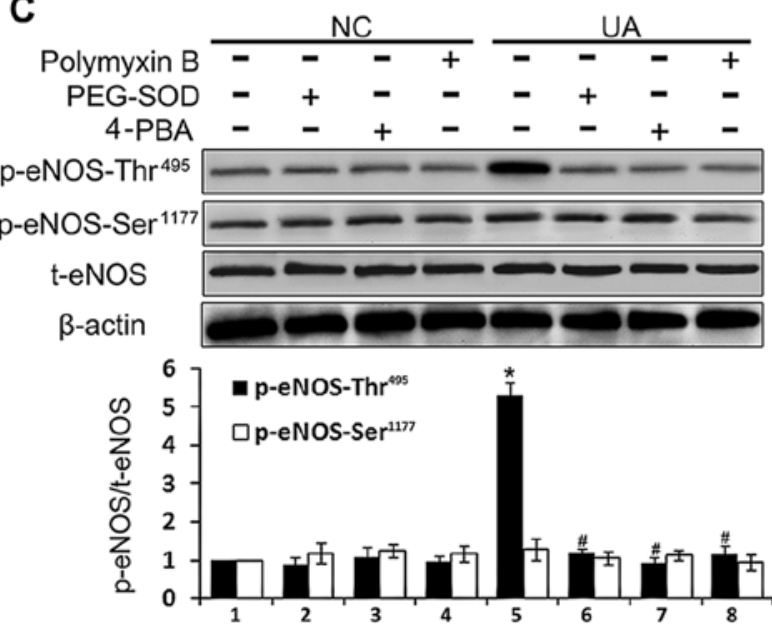

B

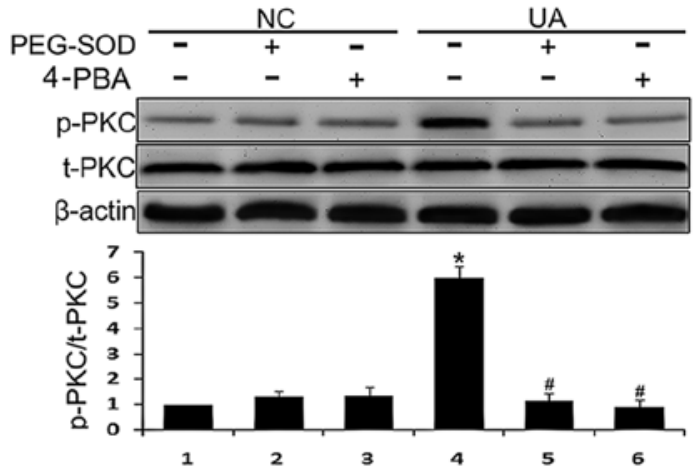

D

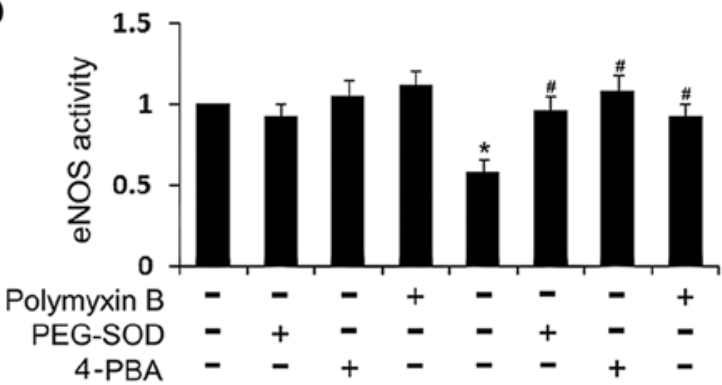

Figure 6. Effect of pharmacological inhibition on the protein kinase C (PKC)/endothelial nitric oxide synthase (eNOS) pathway in uric acid (UA)-stimulated human umbilical vein endothelial cells (HUVECs). NC indicates HUVECs which were incubated without UA stimulation, just with different pharmacological pretreatment. (A) HUVECs were incubated with $12 \mathrm{mg} / \mathrm{dl}$ of UA for the indicated time periods. Cellular content of PKC and p-PKC was assessed by western blot analysis. (B) HUVECs were incubated with $12 \mathrm{mg} / \mathrm{dl}$ of UA with or without a 30-min pre-incubation with polyethylene glycol-superoxide dismutase (PEG-SOD) or 4-phenylbutyric acid (4-PBA). Cellular content of PKC and p-PKC was assessed by western blot analysis. (C) HUVECs were incubated with $12 \mathrm{mg} / \mathrm{dl}$ of UA with or without a 30-min pre-incubation with PEG-SOD, 4-PBA, or polymyxin B. Cellular content of p-eNOS-Ser ${ }^{1177}$, p-eNOS-Thr ${ }^{495}$, and eNOS was assessed by western blot analysis. (D) eNOS activity was detected in HUVECs incubated with $12 \mathrm{mg} / \mathrm{dl}$ UA with or without a 30 -min pre-incubation with PEG-SOD, 4-PBA, or polymyxin B. ${ }^{*} \mathrm{P}<0.05$ vs. control; ${ }^{\#} \mathrm{P}<0.05$ vs. 6 h and UA; $\mathrm{n}=3$. NC, HUVECs not stimulated with UA.

and NO production in a dose- and time-dependent manner. A high concentration of UA induced intracellular ROS accumulation, likely triggering ER stress, and increasing eNOS $\mathrm{Thr}^{495}$ phosphorylation via a PKC-dependent pathway, and reduced eNOS CaM binding and activity. Moreover, we found that pharmacological suppression of oxidative stress and ER stress by PEG-SOD and 4-PBA improved UA-induced apoptosis, eNOS inhibition, and NO production.

Hyperuricemia is a risk factor for the pathogenesis of CVD and CKD (34,35). However, the molecular mechanisms responsible for these observations have not been clearly defined, limiting the development of interventions targeting this process (22). UA has been shown to be involved in oxidative stress through two mechanisms: activation of NADPH oxidase and decrease of NO bioavailability. Indeed, UA participates in CKD through kidney cell apoptosis triggered by the activation of NADPH oxidase (36) and mesangial cell proliferation via the NADPH/ROS/ERK1/1 pathway (37). NADPH oxidase activation by UA also participates in the aging and death of human endothelial cells $(12,38)$. Decreased NO bioavailability is a hallmark of endothelial dysfunction; eNOS, which is required for NO production, is activated by the binding of CaM $(33,39,40)$. UA has previously been reported to reduce NO production in vascular endothelial cells in a dose-dependent manner, possibly through the interaction of eNOS and CaM, although UA did not alter the cellular content of $\mathrm{Ca}^{2+}$ or $\mathrm{CaM}$ in endothelial cells $(22,26)$. Similarly, in the present study, a high concentration of UA did not markedly alter the cellular content of $\mathrm{Ca}^{2+}, \mathrm{CaM}$, or eNOS, but inhibited eNOS binding to CaM.

We also found that UA enhanced eNOS phosphorylation at $\mathrm{Thr}^{495}$, but not at $\mathrm{Ser}^{1177}$. Phosphorylation of eNOS at $\mathrm{Thr}^{495}$ is PKC-dependent, and inhibition of PKC activity reduced UA-induced eNOS phosphorylation at $\mathrm{Thr}^{495}$. Taken together, these findings suggest that in endothelial cells UA activates PKC to phosphorylate eNOS at $\mathrm{Thr}^{495}$. Phosphorylated eNOS cannot bind to CaM (27), and thus remains enzymatically inactive.

Previous research has suggested that UA inhibits eNOS phosphorylation at $\mathrm{Ser}^{1177}$ and NO production in acetylcholine-stimulated HUVECs (23). Additionally, insulin-stimulated eNOS phosphorylation and NO production in vascular endothelial cells was inhibited by UA (41). The discrepancies between our results and these previous results probably lie in the different kinases and downstream signaling pathways which UA affects; acetylcholine and insulin activate the PI3K/ Akt pathway to phosphorylate eNOS at $\operatorname{Ser}^{1177}$, while PKC is 
activated by UA and PKC inactivates eNOS enzymatic activity by phosphorylation at $\mathrm{Thr}^{495}$.

In a murine model of hyperuricemia, serum UA levels were negatively correlated with serum NO and superoxide dismutase levels. In pancreatic $\beta$-cells, UA has previously been reported to induce oxidative stress and growth inhibition by activating the AMP-activated protein kinase (AMPK) signaling pathway (11).

In this study, we noted that in primary cultured HUVECs, UA reduced NO production and induced apoptosis in a dose- and time-dependent manner. UA also induced ROS generation, suggesting a damaging, rather than anti-oxidative, effect, as previously reported elsewhere $(6,9,11)$. We also noted that the antioxidant PEG-SOD significantly inhibited the effect of UA on apoptosis and NO release, suggesting that oxidative stress is key to UA-stimulated endothelial dysfunction.

UA has previously been reported to trigger the ER stress response in rat glomerular mesangial cells in vitro (20). In the present study, we noted that UA upregulated the ER stress markers CHOP and ATF-6, and the ER stress-induced apoptosis marker caspase-12. Elevation of free radicals is one of the factors that trigger ER stress (42). We found that ER stress markers ATF- 6 and CHOP began to significantly increase after $6 \mathrm{~h}$ of UA stimulation, and caspase-12 levels began to significantly increase after $12 \mathrm{~h}$. However, even $3 \mathrm{~h}$ of UA stimulation triggered ROS generation, and inhibition of ROS generation with PEG-SOD attenuated UA-induced ER stress and apoptosis. These findings suggest that UA-stimulated oxidative stress precedes ER stress. ER stress modulates cellular function through the PKC signaling pathway, and knockdown of PKC attenuates ER stress-induced cellular damage (17-19). In this study, we found that in cultured HUVECs, UA triggered PKC phosphorylation in a time-dependent manner. Antioxidant and ER stress inhibitors inhibited UA-induced PKC activation and phosphorylation of eNOS at $\mathrm{Thr}^{495}$, thus preventing UA-mediated depression of $\mathrm{NO}$ production.

In conclusion, the present study confirmed previous reports that high levels of UA trigger endothelial dysfunction, inducing apoptosis and reducing eNOS activity and NO production. We were able to implicate further molecular mechanisms in this process, and hypothesize that UA activates the PKC pathway to trigger oxidative stress and ER stress signaling network to induce endothelial dysfunction in HUVECs. The implications of these mechanisms in vivo remain to be seen, and whether the concentrations of UA employed in this study accurately represent the stresses endothelial cells experience in vivo also remains to be determined.

\section{Acknowledgements}

The study was supported by the Shandong Provincial Science Foundation for Outstanding Young Scientists, China (grant no. BS2013YY051).

\section{References}

1. Erdogan D, Icli A, Aksoy F, Akcay S, Ozaydin M, Ersoy I, Varol E and Dogan A: Relationships of different blood pressure categories to indices of inflammation and platelet activity in sustained hypertensive patients with uncontrolled office blood pressure. Chronobiol Int 30: 973-980, 2013.
2. Kanbay M, Segal M, Afsar B, Kang DH, Rodriguez-Iturbe B and Johnson RJ: The role of uric acid in the pathogenesis of human cardiovascular disease. Heart 99: 759-766, 2013.

3. Storhaug HM, Norvik JV, Toft I, Eriksen BO, Løchen ML, Zykova S, Solbu M, White S, Chadban S and Jenssen T: Uric acid is a risk factor for ischemic stroke and all-cause mortality in the general population: a gender specific analysis from The Troms $\varnothing$ Study. BMC Cardiovasc Disord 13: 115, 2013.

4. Sun Y, Yu X, Zhi Y, Geng S, Li H, Liu T, Xu K, Chen L, Wu C and Qi G: A cross-sectional analysis of the relationship between uric acid and coronary atherosclerosis in patients with suspected coronary artery disease in China. BMC Cardiovasc Disord 14: 101, 2014.

5. Preitner F, Laverriere-Loss A, Metref S, Da Costa A, Moret C, Rotman S, Bazin D, Daudon M, Sandt C, Dessombz A and Thorens B: Urate-induced acute renal failure and chronic inflammation in liver-specific Glut9 knockout mice. Am J Physiol Renal Physiol 305: F786-F795, 2013.

6. Ryu ES, Kim MJ, Shin HS, Jang YH, Choi HS, Jo I, Johnson RJ and Kang DH: Uric acid-induced phenotypic transition of renal tubular cells as a novel mechanism of chronic kidney disease. Am J Physiol Renal Physiol 304: F471-F480, 2013.

7. Glantzounis GK, Tsimoyiannis EC, Kappas AM and Galaris DA: Uric acid and oxidative stress. Curr Pharm Des 11: 4145-4151, 2005.

8. Ames BN, Cathcart R, Schwiers E and Hochstein P: Uric acid provides an antioxidant defense in humans against oxidant- and radical-caused aging and cancer: a hypothesis. Proc Natl Acad Sci USA 78: 6858-6862, 1981.

9. Sánchez-Lozada LG, Lanaspa MA, Cristóbal-García M, García-Arroyo F, Soto V, Cruz-Robles D, Nakagawa T, Yu MA, Kang DH and Johnson RJ: Uric acid-induced endothelial dysfunction is associated with mitochondrial alterations and decreased intracellular ATP concentrations. Nephron Exp Nephrol 121: e71-e78, 2012.

10. Arai Y, Nishinaka Y, Arai T, Morita M, Mizugishi K, Adachi S, Takaori-Kondo A, Watanabe $\mathrm{T}$ and Yamashita K: Uric acid induces NADPH oxidase-independent neutrophil extracellular trap formation. Biochem Biophys Res Commun 443: 556-561, 2014.

11. Zhang Y, Yamamoto T, Hisatome I, Li Y, Cheng W, Sun N, Cai B, Huang T, Zhu Y, Li Z, et al: Uric acid induces oxidative stress and growth inhibition by activating adenosine monophosphate-activated protein kinase and extracellular signal-regulated kinase signal pathways in pancreatic $\beta$ cells. Mol Cell Endocrinol 375: 89-96, 2013.

12. Yu MA, Sánchez-Lozada LG, Johnson RJ and Kang DH: Oxidative stress with an activation of the renin-angiotensin system in human vascular endothelial cells as a novel mechanism of uric acid-induced endothelial dysfunction. J Hypertens 28: 1234-1242, 2010.

13. Oslowski CM and Urano F: Measuring ER stress and the unfolded protein response using mammalian tissue culture system. Methods Enzymol 490: 71-92, 2011.

14. Lenna S, Han R and Trojanowska M: Endoplasmic reticulum stress and endothelial dysfunction. IUBMB Life 66: 530-537, 2014.

15. Chaudhari N, Talwar P, Parimisetty A, Lefebvre d'Hellencourt C and Ravanan P: A molecular web: endoplasmic reticulum stress, inflammation, and oxidative stress. Front Cell Neurosci 8: 213, 2014.

16. Wu CT, Weng TI, Chen LP, Chiang CK and Liu SH: Involvement of caspase-12-dependent apoptotic pathway in ionic radiocontrast urografin-induced renal tubular cell injury. Toxicol Appl Pharmacol 266: 167-175, 2013.

17. Liu M, Zhou L, Wei L, Villarreal R, Yang X, Hu D, Riojas RA, Holmes BM, Langlais PR, Lee H and Dong LQ: Phosphorylation of adaptor protein containing pleckstrin homology domain, phosphotyrosine binding domain, and leucine zipper motif 1 (APPL1) at Ser430 mediates endoplasmic reticulum (ER) stress-induced insulin resistance in hepatocytes. J Biol Chem 287: 26087-26093, 2012.

18. Madaro L, Marrocco V, Carnio S, Sandri M and Bouché M: Intracellular signaling in ER stress-induced autophagy in skeletal muscle cells. FASEB J 27: 1990-2000, 2013.

19. Henstridge DC, Whitham M and Febbraio MA: Chaperoning to the metabolic party: the emerging therapeutic role of heat-shock proteins in obesity and type 2 diabetes. Mol Metab 3: 781-793, 2014. 
20. Li S, Zhao F, Cheng S, Wang X and Hao Y: Uric acid-induced endoplasmic reticulum stress triggers phenotypic change in rat glomerular mesangial cells. Nephrology (Carlton) 18: 682-689, 2013.

21. Choi YJ, Shin HS, Choi HS, Park JW, Jo I, Oh ES, Lee KY, Lee BH, Johnson RJ and Kang DH: Uric acid induces fat accumulation via generation of endoplasmic reticulum stress and SREBP-1c activation in hepatocytes. Lab Invest 94: 1114-1125, 2014.

22. Park JH, Jin YM, Hwang S, Cho DH, Kang DH and Jo I: Uric acid attenuates nitric oxide production by decreasing the interaction between endothelial nitric oxide synthase and calmodulin in human umbilical vein endothelial cells: a mechanism for uric acid-induced cardiovascular disease development. Nitric Oxide 32: 36-42, 2013.

23. Papežíková I, Pekarová M, Kolářová H, Klinke A, Lau D, Baldus S, Lojek A and Kubala L: Uric acid modulates vascular endothelial function through the down regulation of nitric oxide production. Free Radic Res 47: 82-88, 2013.

24. Yelken B, Caliskan Y, Gorgulu N, Altun I, Yilmaz A, Yazici H, Oflaz $\mathrm{H}$ and Yildiz A: Reduction of uric acid levels with allopurinol treatment improves endothelial function in patients with chronic kidney disease. Clin Nephrol 77: 275-282, 2012.

25. Dogan A, Yarlioglues M, Kaya MG, Karadag Z, Dogan S, Ardic I, Dogdu O, Kilinc Y, Zencir C, Akpek M, et al: Effect of long-term and high-dose allopurinol therapy on endothelial function in normotensive diabetic patients. Blood Press 20: 182-187, 2011.

26. Hong Q, Qi K, Feng Z, Huang Z, Cui S, Wang L, Fu B, Ding R, Yang J, Chen Xand Wu D: Hyperuricemia induces endothelial dysfunction via mitochondrial $\mathrm{Na}^{+} / \mathrm{Ca}^{2+}$ exchanger-mediated mitochondrial calcium overload. Cell Calcium 51: 402-410, 2012.

27. Fleming I, Fisslthaler B, Dimmeler S, Kemp BE and Busse R: Phosphorylation of $\mathrm{Thr}(495)$ regulates $\mathrm{Ca}(2+) /$ calmodulin-dependent endothelial nitric oxide synthase activity. Circ Res 88: E68-E75, 2001.

28. Cheng Z, Jiang X, Pansuria M, Fang P, Mai J, Mallilankaraman K, Gandhirajan RK, Eguchi S, Scalia R, Madesh M, et al: Hyperhomocysteinemia and hyperglycemia induce and potentiate endothelial dysfunction via $\mu$-calpain activation. Diabetes 64 947-959, 2015.

29. Han HJ, Lim MJ, Lee YJ, Lee JH, Yang IS and Taub M: Uric acid inhibits renal proximal tubule cell proliferation via at least two signaling pathways involving PKC, MAPK, cPLA2, and NF-kappaB. Am J Physiol Renal Physiol 292: F373-F381, 2007.

30. Jiao K, Zhang J, Zhang M, Wei Y, Wu Y, Qiu ZY, He J, Cao Y, $\mathrm{Hu}$ J, Zhu H, et al: The identification of CD163 expressing phagocytic chondrocytes in joint cartilage and its novel scavenger role in cartilage degradation. PLoS One 8: e53312, 2013.
31. Ding L and Zhang J: Glucagon-like peptide-1 activates endothelial nitric oxide synthase in $\mathrm{h}$ uman umbilical vein endothelial cells. Acta Pharmacol Sin 33: 75-81, 2012.

32. Li P, Zhou C, Yin L, Meng X and Zhang L: Role of hypoxia in viability and endothelial differentiation potential of UC-MSCs and VEGF interference. Zhong Nan Da Xue Xue Bao Yi Xue Ban 38: 329-340, 2013.

33. Xiao Z, Wang T, Qin H, Huang C, Feng Y and Xia Y: Endoplasmic reticulum $\mathrm{Ca}^{2+}$ release modulates endothelial nitric-oxide synthase via extracellular signal-regulated kinase (ERK) 1/2-mediated serine 635 phosphorylation. J Biol Chem 286: 20100-20108, 2011.

34. Zoccali C, Maio R, Mallamaci F, Sesti G and Perticone F: Uric acid and endothelial dysfunction in essential hypertension. J Am Soc Nephrol 17: 1466-1471, 2006.

35. Khosla UM, Zharikov S, Finch JL, Nakagawa T, Roncal C, Mu W, Krotova K, Block ER, Prabhakar S and Johnson RJ: Hyperuricemia induces endothelial dysfunction. Kidney Int 67: 1739-1742, 2005

36. Verzola D, Ratto E, Villaggio B, Parodi EL, Pontremoli R, Garibotto $\mathrm{G}$ and Viazzi F: Uric acid promotes apoptosis in human proximal tubule cells by oxidative stress and the activation of NADPH oxidase NOX 4. PLoS One 9: e115210, 2014.

37. Zhuang Y, Feng Q, Ding G, Zhao M, Che R, Bai M, Bao H, Zhang A and Huang S: Activation of ERK1/2 by NADPH oxidaseoriginated reactive oxygen species mediates uric acid-induced mesangial cell proliferation. Am J Physiol Renal Physiol 307: F396-F406, 2014.

38. Higashi Y, Maruhashi T, Noma K and Kihara Y: Oxidative stress and endothelial dysfunction: clinical evidence and therapeutic implications. Trends Cardiovasc Med 24: 165-169, 2014.

39. Nathan C and Xie QW: Nitric oxide synthases: roles, tolls, and controls. Cell 78: 915-918, 1994.

40. Griffith OW and Stuehr DJ: Nitric oxide synthases: properties and catalytic mechanism. Annu Rev Physiol 57: 707-736, 1995

41. Choi YJ, Yoon Y, Lee KY, Hien TT, Kang KW, Kim KC, Lee J, Lee MY, Lee SM, Kang DH and Lee BH: Uric acid induces endothelial dysfunction by vascular insulin resistance associated with the impairment of nitric oxide synthesis. FASEB J 28: 3197 3204, 2014.

42. Cheng YC, Chen CA, Chang JM and Chen HC: Albumin overload down-regulates integrin- $\beta 1$ through reactive oxygen species-endoplasmic reticulum stress pathway in podocytes. J Biochem 158: 101-108, 2015. 